\title{
REFORMA CATALÍTICA DE METANO PARA REDUÇÃO DIRETA DE FERRO*
}

\author{
Tiago Ramos Ribeiro ${ }^{1}$ \\ João Batista Ferreira Neto ${ }^{1}$ \\ João Guilherme Rocha Poço² \\ Cyro Takano ${ }^{3}$ \\ Leiv Kolbeinsen ${ }^{4}$ \\ Eli Ringdalen ${ }^{5}$
}

\section{Resumo}

Os processos de redução direta utilizam-se de gases redutores $\left(\mathrm{CO}\right.$ e $\left.\mathrm{H}_{2}\right)$ para redução do ferro e produção do DRI. A geração deste gás se dá através de reforma de metano que pode ser feita em um reformador, ou dentro do reator de redução com o DRI como catalizador. O último caso ocorre nos processos auto reformadores. A cinética da reforma de metano com $\mathrm{H}_{2} \mathrm{O}$ catalisada por DRI foi estudada em temperaturas entre $875^{\circ} \mathrm{C}$ e $1050^{\circ} \mathrm{C}$. Os resultados mostraram que até conversão de metano de 0,50 , a reforma é controlada por reação química catalítica conforme mecanismo proposto por Münster e Grabke(3,4). Acima desta conversão, o mecanismo não foi determinado. $O$ uso de gás com potencial abaixo do limite para carburação $\left(\mathrm{CH}_{4} / \mathrm{H}_{2} \mathrm{O}<1\right)$ resultou em DRI sem carbono. Com gás de potencial igual ou acima do limite, a carburação do DRI ocorreu e no caso do gás similar ao do processo industrial a carburação impediu a reação catalítica de reforma.

Palavras-chave: Redução direta; processos auto reformadores; DRI; reforma de metano.

\section{CATALYTIC REFORMING OF METHANE FOR DIRECT REDUCTION OF IRON Abstract}

Direct Reduction processes use gases $\left(\mathrm{CO}\right.$ and $\left.\mathrm{H}_{2}\right)$ for iron reduction and DRI production. The generation of this gas occurs through methane reforming, which can be done in a reformer or inside the reduction shaft with the DRI as catalyst. The latter occurs in the self-reforming processes. The kinetics of steam reforming of methane catalyzed by $\mathrm{DRI}$ was studied at temperatures between $875^{\circ} \mathrm{C}$ and $1050^{\circ} \mathrm{C}$. The results showed that up to methane conversion of 0.50 , reforming is controlled by catalytic chemical reaction according to the mechanism proposed by Münster and Grabke ${ }^{(3,4)}$. Above this point, the mechanism is not clear. Using gas with potential below the limit for carburization $\left(\mathrm{CH}_{4} / \mathrm{H}_{2} \mathrm{O}<1\right)$ resulted in $\mathrm{DRI}$ without carbon. With potential at or above the limit, DRI was carburized and in the case of the gas similar to the industrial process the carburization hindered the catalytic reforming reaction.

Keywords: Direct reduction; self-reforming process; DRI; steam reforming of methane.

1 Laboratório de Processos Metalúrgicos, Centro de Tecnologia em Metalurgia e Materiais, Instituto de Pesquisas Tecnológicas do Estado de São Paulo - IPT, São Paulo, SP, Brasil.tiagorr@ipt.br

2 Departamento de Eng. Química. Centro Universitário FEl, São Bernardo do Campo, SP, Brasil.

3 Departamento de Eng. Metalúrgica e de Materiais, Escola Politécnica da Universidade de São Paulo - USP, São Paulo, SP, Brasil.

4 Faculdade de Ciências Naturais, Universidade Norueguesa de Ciência e Tecnologia - NTNU, Trondheim, Noruega.

5 SINTEF Materiais e Química, Fundação para Pesquisa Científica e Industrial - SINTEF, Trondheim, Noruega. 


\section{INTRODUÇÃO}

O Brasil é um dos grandes exportadores mundiais de minério de ferro. O mercado para pelotas de redução direta demanda um produto de alto teor de ferro contido e consequente maior valor agregado. Desta forma, é necessário conhecer os fundamentos dos processos de redução direta e o comportamento dos diferentes tipos de pelota, a fim de posicionar e precificar melhor o produto.

A produção mundial de ferro-esponja ou DRI (do inglês direct reduced iron) atingiu 72,6 Mt em 2015, demandando aproximadamente $105 \mathrm{Mt}$ de pelotas. 80\% do DRI foi produzido por processos baseados em redução gasosa, principalmente Midrex e Energiron/HyL (1). Tradicionalmente, $\mathrm{CO}$ e $\mathrm{H}_{2}$ são gerados em um reator separado onde gás natural é reformado por reação catalítica com vapor d'água ou $\mathrm{CO}_{2}$. Este gás de redução é então direcionado ao reator de redução do tipo cuba, onde os gases aquecem o leito de pelotas reagindo com os óxidos de ferro.

Um conceito diferente está em uso onde gás natural e vapor d'água são injetados diretamente no reator de redução e a reação de reforma é catalisada pelo DRI. Estes sistemas são denominados Zero Reformer (ZR) ou auto reformadores onde a reforma ocorre in-situ(2). A reforma ocorre nas etapas finais da redução e a carburação do DRI também pode ocorrer.

Neste contexto, o objetivo do presente trabalho é o estudo da cinética da reforma de metano com vapor d'água catalisada pelo DRI.

O diagrama presente na Figura 1 foi desenhado para melhor entendimento das condições de equilíbrio no processo de auto reforma. A fração molar de $\mathrm{C}, \mathrm{O}$ e $\mathrm{H}_{2}$ é calculada conforme mostrado nas equações de 1 a 7 . Este diagrama mostra as regiões em que os diferentes óxidos de ferro estão em equilíbrio com certas misturas gasosas e também a região onde carburação pode ocorrer. Para a faixa de temperatura utilizada neste trabalho a atividade de carbono na cementita $\left(\mathrm{Fe}_{3} \mathrm{C}\right)$ é próxima da unitária e a área de carburação representa as condições termodinâmicas para a formação tanto de carbono sólido como de cementita. Neste diagrama todas as possíveis reações estão consideradas, quais sejam, redução, carburação, reforma, a reação de shift da água e a reação de Boudouard. A composição do gás de redução do processo Energiron/HyL - ZR também está mostrada no diagrama.

Baseado neste diagrama é possível selecionar composições de gases que sejam estáveis com o ferro metálico, de forma a isolar as reações de reforma do metano e evitar a ocorrência de carburação e oxidação do ferro.

$$
\begin{array}{r}
C=\frac{n_{C}}{n_{T}} \\
H_{2}=\frac{n_{H_{2}}}{n_{T}} \\
O=\frac{n_{O}}{n_{T}}
\end{array}
$$

onde,

$$
\begin{array}{r}
n_{\mathrm{C}}=y_{\mathrm{CO}}+y_{\mathrm{CO}_{2}}+y_{\mathrm{CH}_{4}} \\
n_{\mathrm{H}_{2}}=y_{\mathrm{H}_{2}}+y_{\mathrm{H}_{2} \mathrm{O}}+2 \cdot y_{\mathrm{CH}_{4}} \\
n_{\mathrm{O}}=y_{\mathrm{H}_{2} \mathrm{O}}+y_{\mathrm{CO}}+2 \cdot y_{\mathrm{CO}_{2}} \\
n_{\mathrm{T}}=n_{\mathrm{C}}+n_{\mathrm{O}}+n_{\mathrm{H}_{2}}
\end{array}
$$


onde yi é a fração molar do gás i.

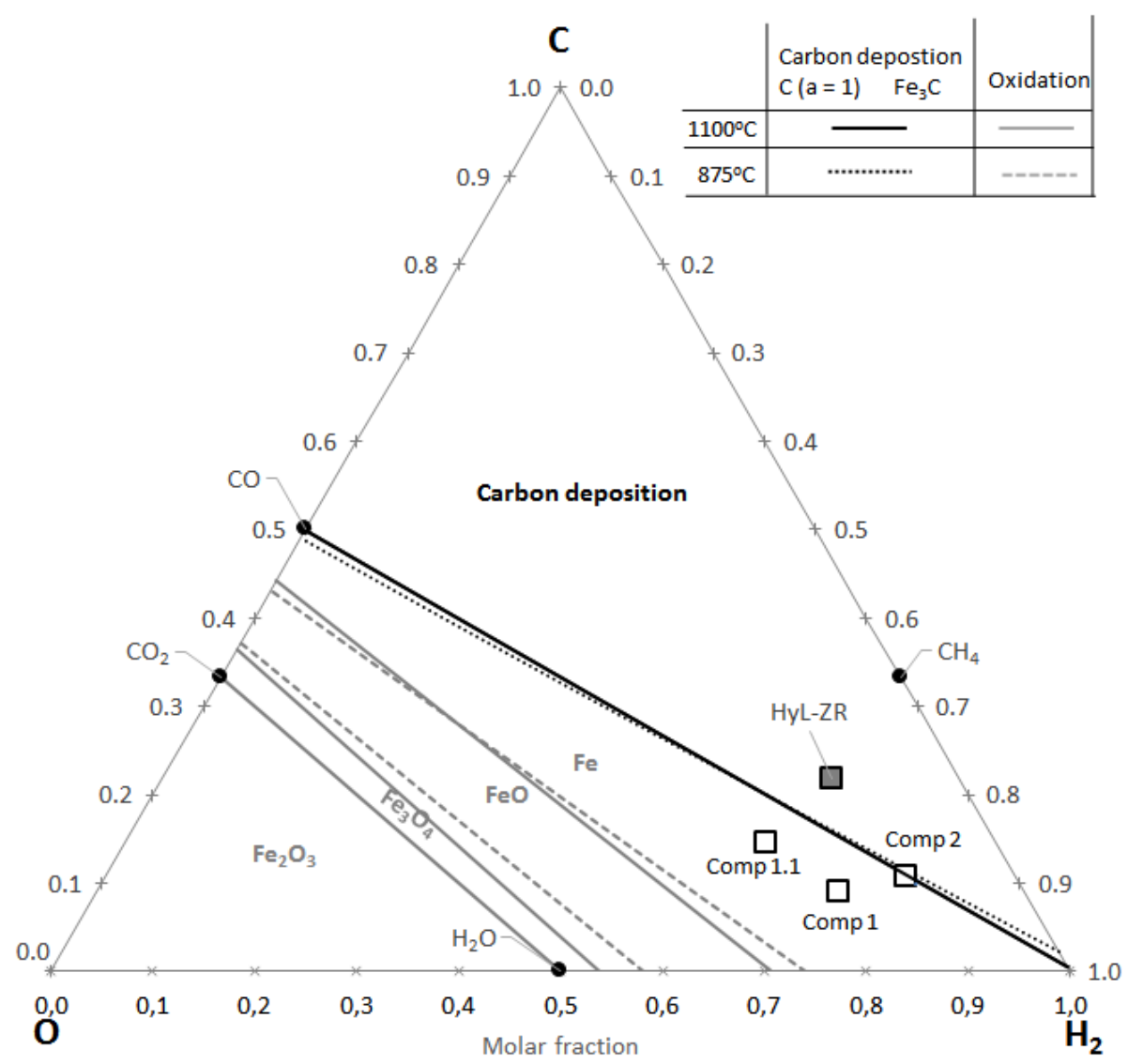

Figura 1. Diagrama C-O- $\mathrm{H}_{2}$ mostrando as áreas de predominância das diferentes fases em equilíbrio com misturas gasosas. Pressão total de $1 \mathrm{~atm}$.

A reforma catalítica de metano com vapor (equação 8) é um tópico amplamente estudado, mas o ferro é usualmente descartado como catalisador pois carbono se deposita facilmente sobre sua superfície.

Münster e Grabke ${ }^{(3,4)}$ estudaram a cinética e o mecanismo da reação de reforma de metano catalisada por uma folha de ferro. Eles descobriram que a reação ocorre em duas etapas. Primeiro o vapor d'água decompõe-se rapidamente na superfície do ferro gerando oxigênio adsorvido e hidrogênio gasoso (equação 9). Logo, metano só pode adsorver e se decompor nos sítios ativos livres de $\mathrm{O}$ adsorvido.

Os autores também mostraram que a reação de decomposição do metano ocorre em etapas sucessivas formando radicais $\mathrm{CH}_{\mathrm{x}}(0 \leq \mathrm{x} \leq 3)$, conforme mostrado na equação 10. A etapa mais lenta deste processo é a decomposição do radical $\mathrm{CH}_{3}$ para $\mathrm{CH}_{2}$. Com base neste mecanismo, foi proposta e testada a equação para cálculo da taxa de reação $\left(-\mathrm{r}_{\mathrm{CH}_{4}}\right)$ mostrada na equação 11.

$$
\begin{array}{r}
\mathrm{CH}_{4}(\mathrm{~g})+\mathrm{H}_{2} \mathrm{O}(\mathrm{g})=\mathrm{CO}(\mathrm{g})+3 \mathrm{H}_{2}(\mathrm{~g}) \\
\mathrm{H}_{2} \mathrm{O}(\mathrm{g})=\mathrm{O}(\mathrm{ads})+\mathrm{H}_{2}(\mathrm{~g}) \\
\mathrm{CH}_{\mathrm{x}+1}(\mathrm{ads})=\mathrm{CH}_{\mathrm{x}}(\mathrm{ads})+1 / 2 \mathrm{H}_{2}(\mathrm{~g}) \\
-r_{\mathrm{CH}_{4}}=k \cdot \frac{1}{1+K p \mathrm{H}_{2} \mathrm{O} / p \mathrm{H}_{2}} \cdot \frac{p C H_{4}}{p H_{2}{ }^{1 / 2}}
\end{array}
$$


onde, pi é a pressão parcial do gás i, k é a constante cinética e $\mathrm{K}$ é a constante de equilíbrio da reação de decomposição do $\mathrm{H}_{2} \mathrm{O}$ (equação 9).

\section{MATERIAIS E MÉTODOS}

Para realização dos experimentos foi utilizada uma pelota para redução direta produzida pela Vale, cuja composição química está mostrada na Tabela 1.

Tabela 1. Composição química da pelota de redução direta Vale. Valores em \% em massa.

\begin{tabular}{|l|l|l|l|l|l|l|l|l|l|}
\hline Fe total & $\mathrm{SiO}_{2}$ & $\mathrm{Al}_{2} \mathrm{O}_{3}$ & $\mathrm{P}$ & $\mathrm{Mn}$ & $\mathrm{CaO}$ & $\mathrm{MgO}$ & $\mathrm{TiO}_{2}$ & $\mathrm{PF}^{*}$ & $\mathrm{CaO} / \mathrm{SiO}_{2}$ \\
\hline 66,8 & 1,6 & 0,5 & 0,03 & 0,08 & 1,8 & 0,3 & 0,04 & 0,1 & 1,17 \\
\hline
\end{tabular}

O sistema experimental utilizado está mostrado esquematicamente na Figura 2 - a. As vazões dos gases utilizados foram controladas com controladores digitais de fluxo de massa (CFM). Os gases misturados eram injetados no reator que estava posicionado dentro de um forno de resistências e pendurado em uma balança para medida contínua da massa do sistema. O reator metálico construído em aço inoxidável para alta temperatura 253MA (Figura 2 - b) era uma retorta de dupla câmara, onde o gás de entrada era injetado na câmara exterior e fluía para baixo sendo aquecido pelo contato com as paredes. Ao atingir o fundo do reator o gás passava a fluir para cima atravessando a placa de distribuição onde as pelotas ou DRI estavam apoiados. Um termopar tipo S estava posicionado dentro da amostra.

Os gases de saída foram direcionados para um condensador resfriado a água e após por um leito de sílica gel para retenção de vapor d'água. Os gases secos eram analisados quanto aos teores de $\mathrm{CO}$ e $\mathrm{CO}_{2}$ por um analisador infravermelho da marca ABB modelo AO2020.

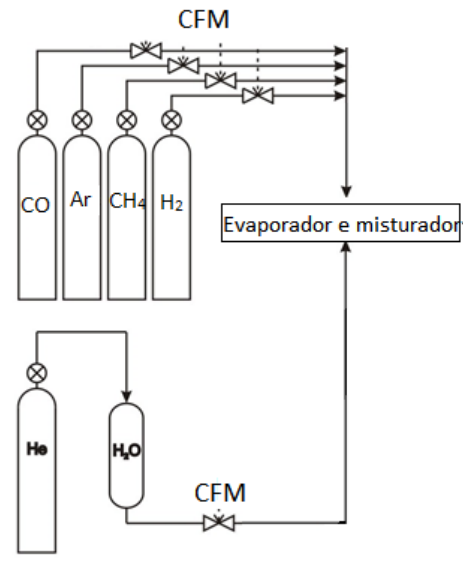

(a)

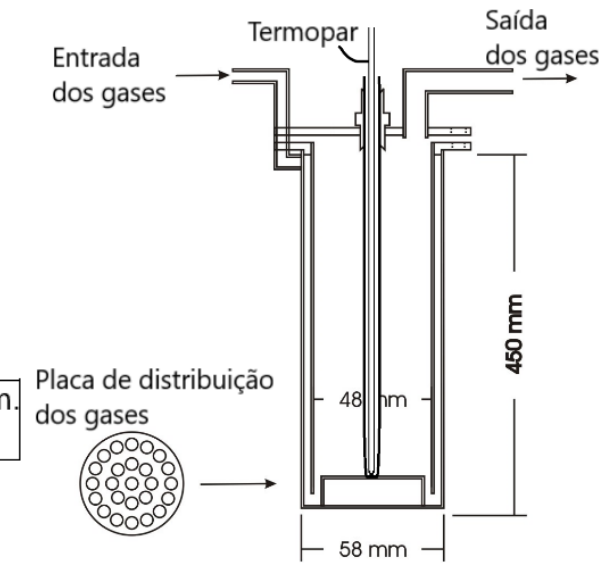

(b)

Figura 2. Desenho esquemático (a) da montagem experimental e (b) do reator utilizados nos experimentos de redução de pelota de minério de reforma de metano catalisada por DRI.

A redução das pelotas de minério de ferro foi realizada previamente, utilizando o mesmo reator. Para a redução utilizou-se $200 \mathrm{~g}$ de pelotas. O sistema foi aquecido até $900^{\circ} \mathrm{C}$ no termopar do forno a $10^{\circ} \mathrm{C} / \mathrm{min}$. Nesta temperatura, iniciou-se a injeção de $5 \mathrm{~L} / \mathrm{min}\left(20^{\circ} \mathrm{C} \text { e } 1 \mathrm{~atm}\right)^{1}$ de hidrogênio por 2 horas. Durante o aquecimento e resfriamento das amostras um fluxo de $1 \mathrm{~L} / \mathrm{min}$ de argônio foi utilizado para manter uma atmosfera inerte.

\footnotetext{
${ }^{1}$ Todas as vazões colocadas neste trabalho se referem a temperatura de $20{ }^{\circ} \mathrm{C}$ e pressão de 1 atmosfera.
} 
A fração de redução $\left(F_{R}\right)$ da pelota foi calculada pela quantidade de oxigênio removida medida pela perda de massa do sistema, em relação à quantidade de oxigênio ligado ao ferro da amostra, considerando que todo o ferro estava na forma de $\mathrm{Fe}_{2} \mathrm{O}_{3}$. Este $\mathrm{DRI}$ resultante da redução das pelotas foi utilizado como matériaprima para os ensaios de reforma de metano.

Os parâmetros dos ensaios de reforma de metano estão mostrados na Tabela 2. As composições dos gases de entrada estão colocadas também na Figura 1.

Tabela 2. Parâmetros dos ensaios de reforma de metano catalisada por DRI.

\begin{tabular}{|c|c|c|c|c|c|c|c|c|}
\hline \multirow[t]{2}{*}{ Comp. } & \multicolumn{5}{|c|}{ vol \% } & \multirow{2}{*}{$\begin{array}{l}\text { Temperatura } \\
\left({ }^{\circ} \mathrm{C}\right)\end{array}$} & \multirow{2}{*}{$\begin{array}{l}\text { Massa de DRI } \\
\text { (g) }\end{array}$} & \multirow{2}{*}{$\begin{array}{l}\text { Vazão total de entrada } \\
\left.\text { (L/min a } 20^{\circ} \mathrm{C} \text { e } 1 \mathrm{~atm}\right)\end{array}$} \\
\hline & $\mathrm{H}_{2}$ & $\mathrm{CH}_{4}$ & $\mathrm{H}_{2} \mathrm{O}$ & $\mathrm{Ar}$ & $\mathrm{CO}$ & & & \\
\hline 1 & 52 & 13 & 26 & 10 & 0 & $\begin{array}{l}875,900,950 \\
1000,1050\end{array}$ & $\begin{array}{l}25,50,65,85 \\
100\end{array}$ & 2,5 a 8,0 \\
\hline 2 & 62 & 14 & 14 & 10 & 0 & $875,950,1050$ & 25 e 50 & 2,5 a 12,0 \\
\hline 3 & 54 & 18 & 5 & 10 & 14 & & & \\
\hline
\end{tabular}

A composição 1 não possui potencial termodinâmico para oxidação ou carburação do DRI, uma vez que a relação $\mathrm{pCH}_{4} / \mathrm{pH}_{2} \mathrm{O}=0,5$ e a relação $\mathrm{pH}_{2} / \mathrm{pH}_{2} \mathrm{O}=2$. A composição 2 é mais redutora com $\mathrm{pCH}_{4} / \mathrm{pH}_{2} \mathrm{O}=1$ e $\mathrm{pH}_{2} / \mathrm{pH}_{2} \mathrm{O}=4,4$. Neste caso, a mistura gasosa está no limite termodinâmico para carburação do ferro nas temperaturas dos ensaios, como pode ser visto na Figura 1. A composição 3 é a composição do gás de redução do processo HyL/Energiron ZR que já tem potencial termodinâmico para carburar o ferro.

As temperaturas utilizadas variaram de $875^{\circ} \mathrm{C}$ até $1050^{\circ} \mathrm{C}$, sendo esta última dentro da faixa de operação do reator industrial. A massa de DRI e vazão de gases de entrada foram alterados nos níveis indicados na Erro! Fonte de referência não encontrada.. Em maiores temperaturas foram utilizadas menores massas de DRI, uma vez que maiores conversões de metano eram esperadas.

$O$ procedimento de ensaio iniciava-se com o aquecimento do sistema a $10^{\circ} \mathrm{C} / \mathrm{min}$ até a temperatura de ensaio. Então iniciava-se a injeção da mistura de gases a ser reformada. A temperatura do forno era continuamente alterada para manter a temperatura do leito de $\mathrm{DRI}$ dentro de um intervalo de $\pm 5^{\circ} \mathrm{C}$ a partir da temperatura desejada. Após estabilização da temperatura e das leituras de $\mathrm{CO}$ e $\mathrm{CO}_{2}$ nos gases de saída, o sistema era mantido constante por pelo menos 15 minutos e até no máximo 40 minutos. Após este período, a vazão de entrada era então alterada e novamente a temperatura do leito necessitava ser ajustada. Este ciclo era repetido até que toda a faixa de vazões mostrada na Tabela 2 fosse coberta em um experimento.

O teor de carbono das amostras de DRI após a redução e após os ensaios de reforma foram analisados por combustão em equipamento da marca LECO.

\section{RESULTADOS E DISCUSSÃO}

Os resultados de redução das pelotas de minério de ferro para produção dos DRIs serão apresentados primeiramente, seguidos dos resultados dos ensaios de reforma e sua interpretação de acordo com o modelo cinético utilizado.

\subsection{Redução das Pelotas de Minério de Ferro}

Os ensaios de redução foram realizados com hidrogênio gasoso para produzir um DRI sem a presença de carbono e, portanto, não interferir nas propriedades catalíticas do ferro reduzido. Foram realizados 24 ensaios de redução ao todo. As curvas da fração de redução com o tempo são similares com reprodutibilidade entre 
os ensaios. A fração de redução final para todos os ensaios conduzidos ficou dentro do intervalo de 0,955 $\pm 0,005$.

A temperatura medida no leito de pelotas variou durante o ensaio, principalmente no início devido as maiores taxas de reação e ao fato de a redução $c o m \mathrm{H}_{2}$ ser endotérmica. A Figura 3 mostra as temperaturas durante o aquecimento e durante 0 período de redução juntamente com a fração de redução de um dos ensaios de redução. A temperatura no leito de pelotas no início do período de redução estava em $940{ }^{\circ} \mathrm{C}$ e em seguida caiu abaixo de $900{ }^{\circ} \mathrm{C}$. A temperatura voltou ao patamar de $940{ }^{\circ} \mathrm{C}$ após a fração de redução atingir o valor de 0,40. Este mesmo comportamento foi observado nos outros ensaios de redução, com variações de magnitude semelhante. A temperatura do forno foi sempre mantida a $900{ }^{\circ} \mathrm{C}$ durante o período de redução.

Os teores de carbono medidos por combustão das amostras reduzidas em dois ensaios distintos foram de $0,02 \%$ e $0,03 \%$, mostrando a ausência de carbono no $\mathrm{DRI}$, conforme desejado.

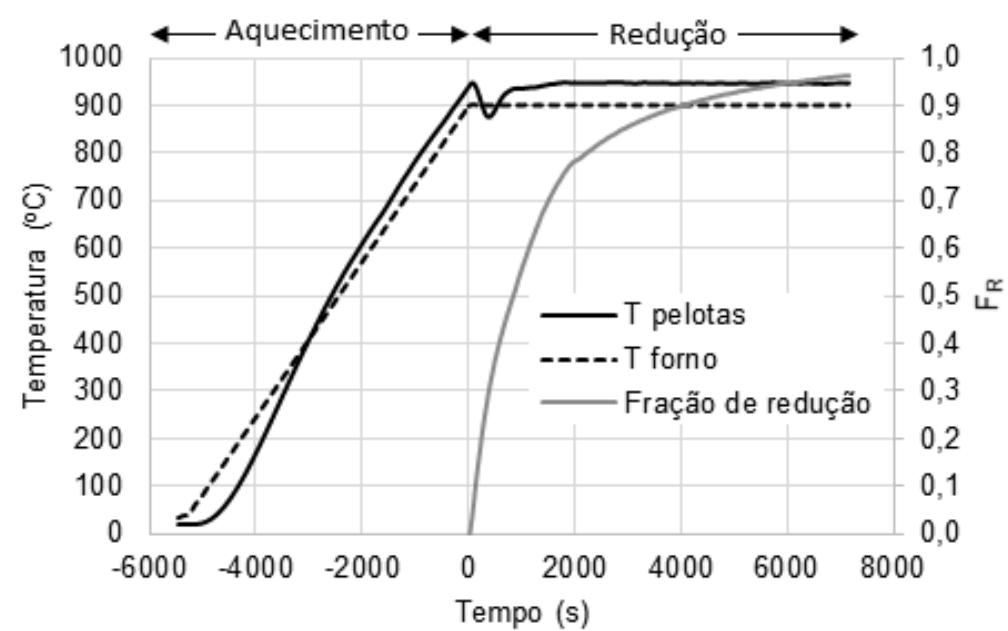

Figura 3. Perfil de temperaturas de um dos ensaios de redução mostrando a temperatura no leito de pelotas e a temperatura do forno durante o aquecimento (tempo <0) e durante a redução (tempo $\geq 0$ ).

\subsection{Reforma catalítica de metano}

Os dados medidos durante os ensaios de reforma de metano foram a temperatura dentro do leito, a massa do sistema de ensaio, as vazões dos gases de entrada e a composição do gás de saída em termos dos teores de $\mathrm{CO}_{\text {e }} \mathrm{CO}_{2}$.

Para o cálculo da conversão de metano, foi realizado um balanço de carbono entre o gás de entrada e o gás de saída, considerando que não haveria deposição de carbono no DRI. A equação 12 mostra este balanço. O fluxo de entrada de metano $\left(\mathrm{F}_{\mathrm{CH}_{4}}\right)$ era controlado pelos MFCs, o fluxo de saída de $\mathrm{CO}(\mathrm{FCO})$ e $\mathrm{CO}_{2}\left(\mathrm{~F}_{\mathrm{CO}_{2}}\right)$ calculados a partir da análise dos gases de saída e fluxo de saída de metano $\left(\mathrm{F}_{\mathrm{CH}_{4}}\right)$ era obtido a partir da equação 12 .

$$
C_{\text {entrada }}=C_{\text {saida }} \rightarrow F_{\mathrm{CH}_{40}}=F_{C O}+F_{\mathrm{CO}_{2}}+F_{\mathrm{CH}_{4}}
$$

O reator utilizado para os ensaios de reforma de metano pode ser descrito por um reator catalítico de leito fixo preenchido com partículas de DRI. Neste reator existe um fluxo de entrada de gases que vão reagindo ao longo do reator e as partículas 
sólidas no interior atuam como catalizadores desta reação. As diferentes posições ao longo do reator são referenciadas pela massa de catalisador presente entre a entrada do reator $(\mathrm{W})$ e o posição em questão.

O balanço de massa deste tipo de reator resulta na equação 13, onde a derivada da conversão de metano $(\mathrm{X})$ em relação à fração $\mathrm{W} / \mathrm{F}_{\mathrm{CH}_{4}}$ é igual a taxa de reação ($\left.\mathrm{rCH}_{4}\right)^{(5,6)}$. Está também mostrado o balanço na forma integral.

$$
\frac{d X}{d\left(W / F_{C H_{4}}\right)}=-r_{C_{4}} \text { ou } \quad \int_{0}^{X} \frac{d X}{-r_{C H_{4}}}=\frac{W}{F_{C H_{40}}}
$$

Os experimentos foram planejados para variar tanto a massa de $\mathrm{DRI}(\mathrm{W})$ quanto a vazão de entrada do metano $\left(\mathrm{F}_{\mathrm{CH}_{4}}\right)$, de forma a varrer uma faixa de valores de conversão do metano.

A Figura 4 mostra os resultados dos experimentos de reforma de metano para as composições 1 e 2, a diferentes temperaturas.

Os resultados mostraram que para maiores temperaturas as conversões são maiores, conforme esperado. Além disso, a taxa de reação é maior no início e a reação se torna mais lenta com o aumento da conversão. Nas mesmas condições (temperatura, massa de DRI e vazão de entrada de metano) as conversões obtidas com a composição 2 são maiores que para a composição 1, indicando que 0 aumento do potencial redutor dos gases melhora a cinética das reações de reforma.

Os resultados obtidos nos experimentos com diferentes massas de DRI para uma mesma temperatura se mostraram concordantes, mantendo as mesmas tendências. Adicionalmente, pontos com diferentes massas de DRI e mesmo valores de W/F $\mathrm{CH}_{4}$ resultaram em conversões de metano similares, com diferenças esperadas pelas variabilidades inerentes ao método experimental.

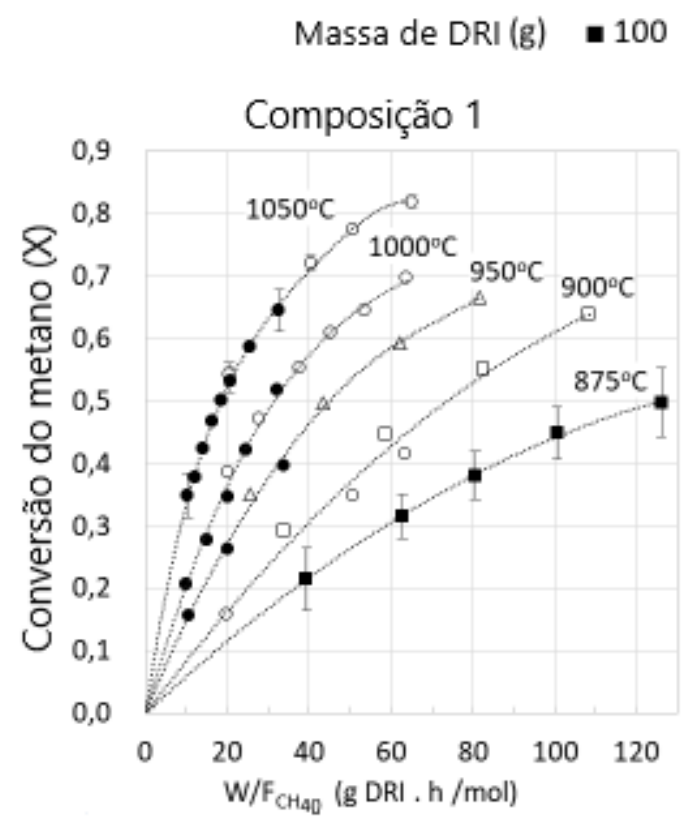

(a)
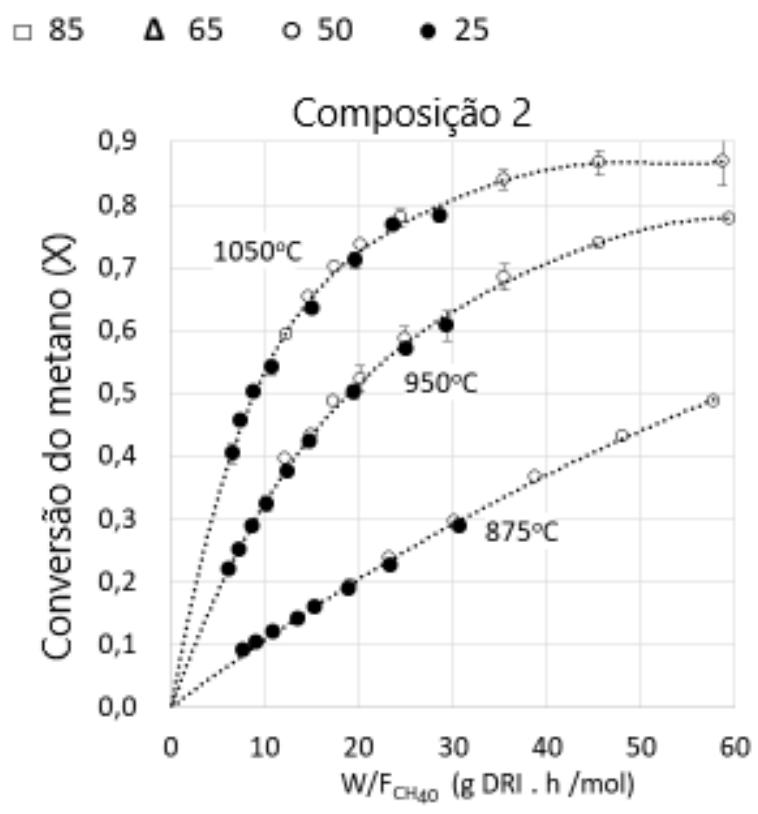

(b)

Figura 4. Resultados de conversão de metano na reação de reforma para a composição dos gases de entrada (a) 1 e (b) 2, a diferentes temperaturas indicadas em cada curva e diferentes massas de 
DRI indicadas pelo símbolo dos pontos. As barras de erro mostradas representam o intervalo de confiança com $95 \%$ de grau de confiança considerando uma distribuição normal.

A partir dos dados levantados, passa-se a análise cinética dos mesmos. Para tal, serão consideradas as seguintes etapas para a reação de reforma do metano catalisada por DRI: (i) difusão dos reagentes pela camada limite gasosa, (ii) difusão dos reagentes pelos poros do DRI, (iii) reação química na superfície interna do DRI, (iv) difusão dos produtos pelos poros do DRI e (v) difusão dos produtos pela camada limite gasosa. A consideração de cada uma destas etapas influenciará na maneira de cálculo da taxa de reação $\left(-\mathrm{rCH}_{4}\right)$ de metano da equação 13.

Em trabalho anterior ${ }^{(6)}$, os autores mostraram que as etapas relacionadas ao transporte de massa dos reagentes e produtos (etapas i, ii, iv e v) não tem influência significativa na cinética da reforma do metano catalisada por DRI. Nestes casos, foram ensaiados DRIs de diâmetros diferentes $(9 \mathrm{~mm}, 12,6 \mathrm{~mm}$ e $17 \mathrm{~mm})$ e as curvas de conversão de metano não mostraram diferenças que justificassem 0 controle por difusão por poros. Para a difusão pela camada limite gasosa, foram analisados resultados obtidos com diferentes velocidades do gás ao redor do $\mathrm{DRI}, 0$ que causaria alterações na espessura da camada gasosa estagnada. E nestes casos as conversões de metano obtidas também mostraram não sofrer influência da difusão por camada limite gasosa.

A partir disto, passa-se a avaliação pelo controle por reação química na interface (etapa iii). O mecanismo proposto por Münster e $\operatorname{Grabke}^{(3,4)}$ para a reação de reforma de metano será a base para a avaliação do controle cinético por reação química. A equação do balanço de massa do reator na forma integral (equação 13) foi combinada com a equação da taxa de reação proposta pelos autores (equação 11). Esta combinação, resultou na equação 14.

$$
\int_{0}^{X} \frac{d X}{\frac{1}{1+K p H_{2} O / p H_{2}} \cdot \frac{p C H_{4}}{p H_{2}{ }^{1 / 2}}}=k \cdot \frac{W}{F_{C_{4} 0}} \quad \text { ou } \quad \int_{0}^{X} \frac{d X}{f(x)}=k \cdot \frac{W}{F_{C H_{4}}}
$$

Onde, $f(X)=\frac{1}{1+\mathrm{KpH}_{2} \mathrm{O} / \mathrm{pH}_{2}} \cdot \frac{p \mathrm{CH}_{4}}{\mathrm{pH}_{2}{ }^{1 / 2}}$.

Aplicando a equação 14 aos dados obtidos para a composição 1 (Figura 4 - a) obtém-se as curvas da Figura 5. Observou-se que até conversões iguais a 0,50 as curvas apresentavam comportamento linear para os dados de todas as temperaturas indicando a validade da equação 14. Acima deste valor de conversão, o comportamento dos dados se alterava. Quase todos os dados obtidos a menores temperaturas $\left(875{ }^{\circ} \mathrm{C}\right.$ e $\left.900{ }^{\circ} \mathrm{C}\right)$ tiveram um bom ajuste a uma reta pois as conversões máximas obtidas foram próximas ou inferiores 0,50 .

É possível fazer a mesma análise com os dados da composição 2. A Figura 6 mostra a aplicação do modelo de controle por reação química nos dados da composição 1 até conversão de 0,50 (pontos fechados) juntamente com os dados experimentais da composição 2 (pontos abertos). Para temperatura de $875{ }^{\circ} \mathrm{C}$, todos os dados obtidos com a composição 2 são de conversões menores que 0,50 e se adequam muito bem a reta obtida com os dados da composição 1 . Para $950{ }^{\circ} \mathrm{C}$ e $1050{ }^{\circ} \mathrm{C}$ uma parte dos dados se adequa bem, e a partir de um determinado ponto os dados passam a ter comportamento distinto das retas obtidas com os dados da composição 1. Este ponto de transição de comportamento, indicado pelas flechas na 
Figura 6, é a conversão de metano de 0,50 para ambas as temperaturas, ou seja, o mesmo valor observado para a composição 1.

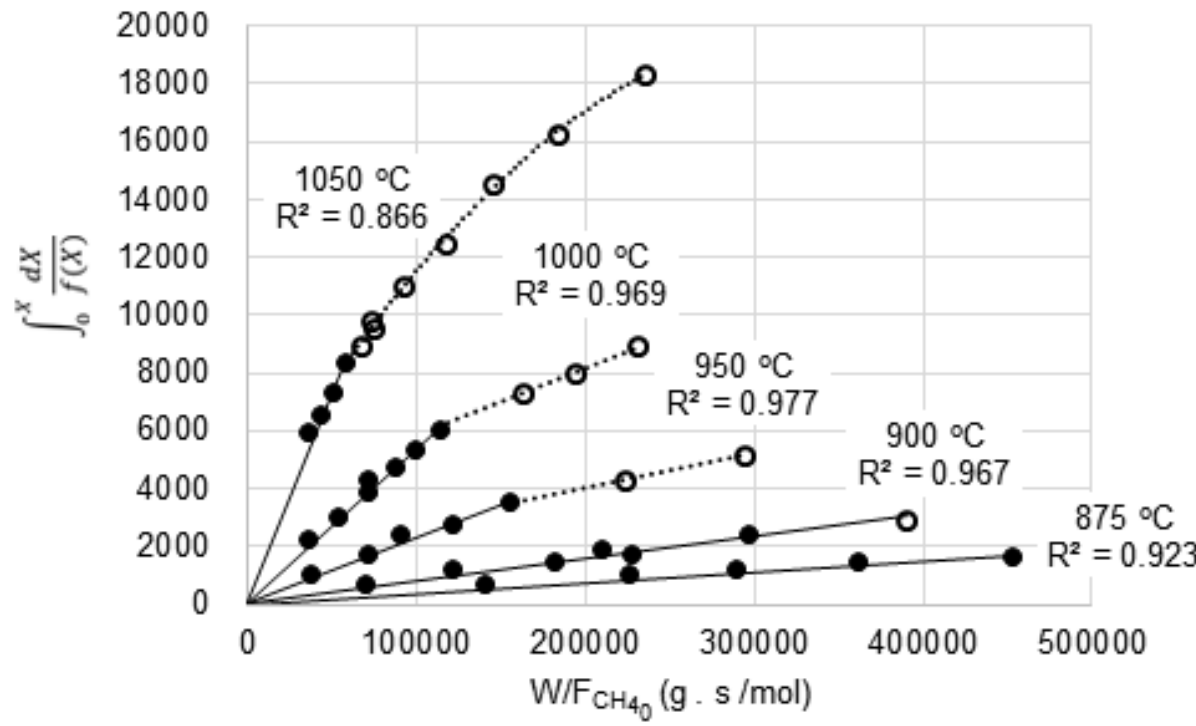

Figura 5. Aplicação do modelo de controle por reação química (equação 14) aos dados obtidos nos experimentos de reforma de metano com composição 1 de gás de entrada até conversão de metano igual a 0,5 (pontos fechados).

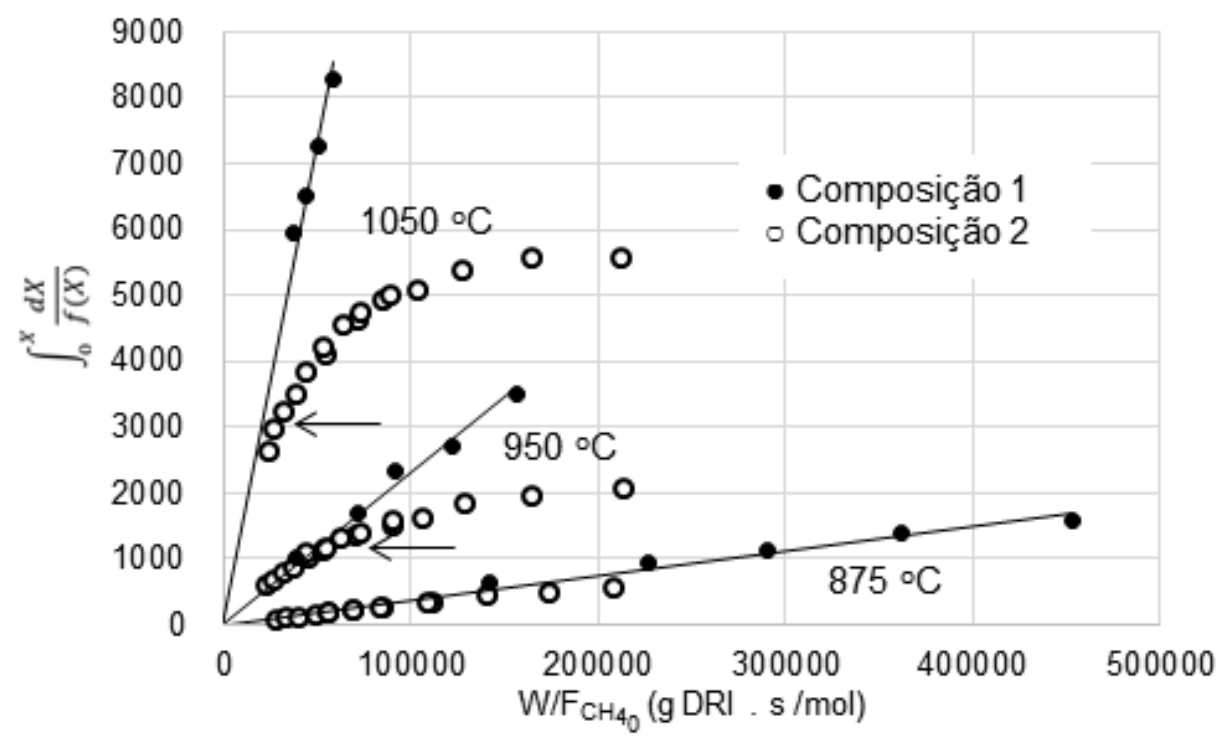

Figura 6. Aplicação do modelo de controle por reação química (equação 14) aos dados obtidos nos experimentos de reforma de metano com composição 1 de gás de entrada até conversão de metano igual a 0,5 (pontos fechados) e composição 2 (pontos abertos).

A partir do coeficiente angular retas obtidas com os dados das composições 1 e 2 é possível obter os valores das constantes cinéticas, conforme mostrado na equação 14. Estas constantes cinéticas estão plotadas no gráfico de Arrhenius da Figura 7. Os dados apresentaram comportamento linear com bom ajuste, o que é mais uma indicação de que é o mesmo mecanismo que está controlando a reforma do metano em todas as temperaturas até conversões de 0,50 . A energia de ativação obtida foi de $258 \mathrm{~kJ} / \mathbf{m o l}$ que corresponde ao controle por reação química catalítica. Além disso, o valor obtido é da mesma ordem de magnitude da energia de ativação obtida a partir dos dados de Münster e Grabke ${ }^{(3,4)}$ de $272 \mathrm{~kJ} / \mathrm{mol}$. 


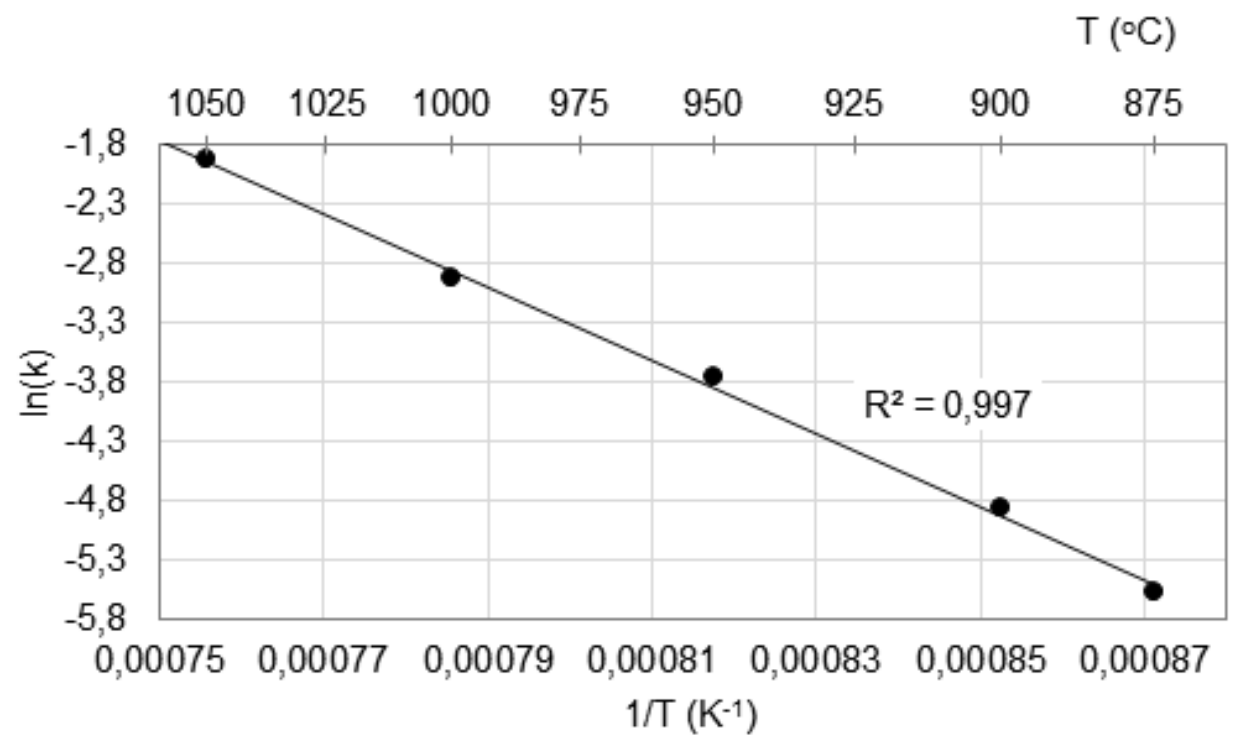

Figura 7. Gráfico de Arrhenius obtido com as constantes cinéticas da reação de reforma de metano.

A análise cinética conduzida até este ponto mostrou que o mecanismo de reação química proposto por Münster e Grabke ${ }^{(3,4)}$ para a reforma de metano catalisada por ferro explicou bem os dados obtidos entre temperaturas de $875^{\circ} \mathrm{C}$ e $1050{ }^{\circ} \mathrm{C}$ até conversões de metano de 0,50, utilizando duas composições de gás com diferentes potenciais redutores.

$\mathrm{Na}$ tentativa de explicar o comportamento dos dados para conversões acima de 0,50 , foram realizados experimentos com a adição de $\mathrm{CO}$ no gás de entrada para verificar a existência de alguma etapa de reação que estivesse próxima do equilíbrio. Logo, a velocidade de reação reduziria pela adição de um produto. No entanto as diferenças observadas não foram significativas. Outra tentativa foi realizada pela utilização de uma pelota de minério de ferro de outro produtor industrial para verificar se os sítios ativos do DRI poderiam ter algum comportamento diferente ao longo da reação. Novamente os resultados não mostraram diferenças significativas. Estes resultados foram publicados com maiores detalhes em outro trabalho dos autores ${ }^{(6)}$. Uma terceira hipótese para explicar o comportamento dos dados experimentais em conversões de metano acima de 0,50 é a carburação do DRI pelo contato com o gás contendo $\mathrm{CH}_{4}$. A carburação pode ter dois efeitos nos resultados obtidos de reforma de metano. O primeiro é de alteração na conversão de metano calculada. A decomposição do metano geraria hidrogênio gasoso que não seria detectado, uma vez que no gás de saída só eram analisados os teores de $\mathrm{CO}$ e $\mathrm{CO}_{2}$. O segundo efeito decorre da possibilidade de o carbono depositado alterar as propriedades da superfície do ferro metálico levando a alteração do seu comportamento catalítico.

Para avaliar tais efeitos, ensaios de reforma foram interrompidos após diferentes intervalos de tempo para medir o teor de carbono depositado no DRI. Estes ensaios foram conduzidos com as composições de entrada 1 e 2 e vazão de entrada de $2,5 \mathrm{~L} / \mathrm{min}$ para ambas. A massa de DRI utilizada foi de $25 \mathrm{~g}$ e $50 \mathrm{~g}$ e a temperatura dos ensaios foi de $1050{ }^{\circ} \mathrm{C}$. Nestas condições a conversão de metano pela reação de reforma era maior do que 0,50 . Os resultados obtidos em termos do teor de carbono no DRI estão mostrados na Figura 8. 


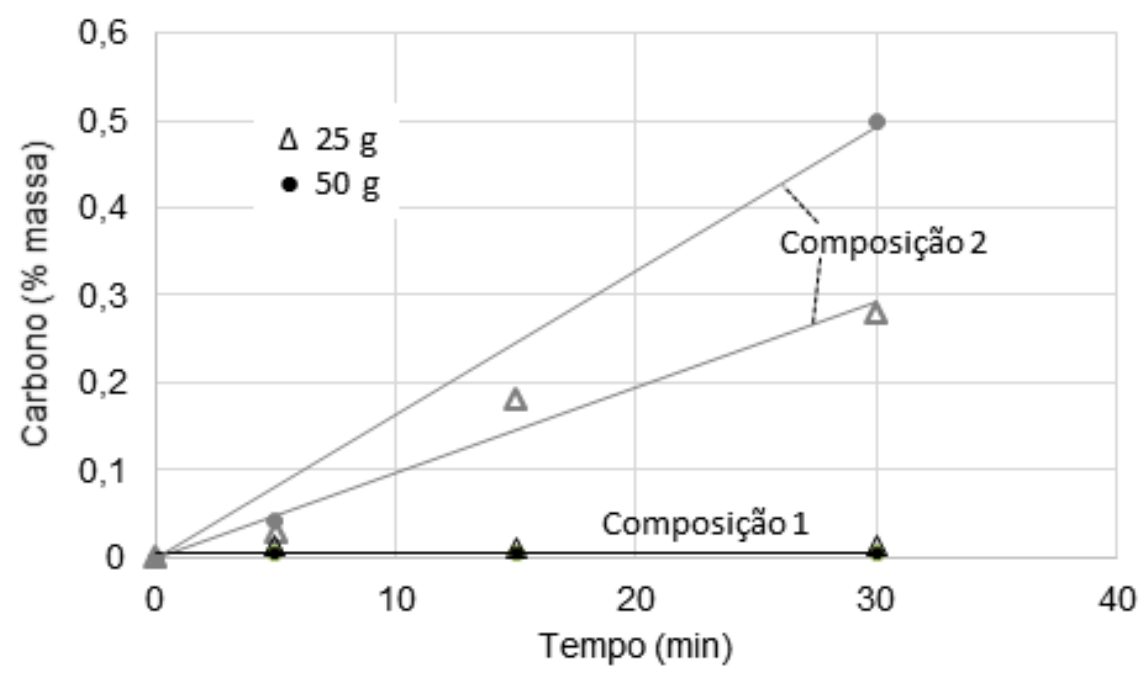

Figura 8. Variação do teor de carbono com o tempo de ensaios de reforma de metano com composições de gás de entrada 1 e 2 , temperatura de $1050^{\circ} \mathrm{C}$, vazão de entrada de $2,5 \mathrm{~L} / \mathrm{min}$ e massa de DRI de $25 \mathrm{~g}$ e $50 \mathrm{~g}$.

Os ensaios com composição 1 não mostraram deposição de carbono, tanto para 25 $\mathrm{g}$ quanto para $50 \mathrm{~g}$ de DRI, uma vez que a composição gasosa não tem potencial termodinâmico para carburar o DRI.

Para a composição 2 do gás, a taxa de carburação dos ensaios com $25 \mathrm{~g}$ de DRI foi menor que dos ensaios com $50 \mathrm{~g}$ de DRI. Isto é uma indicação de que a velocidade da reação de carburação é influenciada pela área de superfície disponível para interação com o gás. Estas taxas medidas correspondem a 1,3\% do metano injetado no gás de entrada nos ensaios com $25 \mathrm{~g}$ e a 2,0\% nos ensaios com $50 \mathrm{~g}$. Logo, esta carburação não influencia significativamente no cálculo da conversão do metano.

O carbono depositado pode ainda alterar as propriedades catalíticas do DRI ao cobrir sítios ativos ou pela formação de cementita. Estas questões serão avaliadas na continuidade do trabalho através de análises de difração de raios- $X$ para determinação das fases presentes e observações microestruturais com microscópio eletrônico. Os resultados serão publicados oportunamente.

Portanto, não foi determinado o mecanismo de controle da reação de reforma em conversões de metano acima de 0,50.

Por último foram realizados os experimentos com a composição 3 que é a composição do processo industrial Energiron/HyL ZR. Esta composição tem potencial termodinâmico para carburar o ferro, como mostrado no diagrama de equilíbrio da Figura 1. Neste caso os resultados obtidos foram diferentes dos obtidos para as demais composições. A conversão de metano foi aproximadamente constante, variando entre 0,08 e 0,12 independentemente dos níveis da relação $\mathrm{W} / \mathrm{F}_{\mathrm{CH}_{4}}$. Este comportamento mostra que a reação deixou de ser catalisada pelo DRI. Além disso, a massa do sistema passou a aumentar com o tempo de ensaio, indicando a deposição de material no DRI.

Os resultados de ensaios interrompidos com a composição 3 mostraram que a taxa de carburação foi intensa com o teor de carbono do DRI atingindo quase $20 \%$ em massa com 30 minutos de reação. Este teor de carbono está acima do teor na cementita $\left(\mathrm{Fe}_{3} \mathrm{C}\right)$, o que mostra que há necessariamente a presença de carbono sólido depositado no DRI. 
A taxa de carburação observada corresponde a $30 \%$ do metano injetado no gás de entrada e influencia diretamente nas medidas das conversões via reação de reforma. Nestas condições, o vapor deixa de reagir com o metano e passa a reagir com o carbono sólido depositado, conforme a reação mostrada na equação 15. Logo, não há uma reação catalítica, mas uma reação gás/sólido.

$$
\mathrm{C}(\mathrm{s})+\mathrm{H}_{2} \mathrm{O}(\mathrm{g})=\mathrm{CO}(\mathrm{g})+\mathrm{H}_{2}(\mathrm{~g})
$$

\section{CONCLUSÃO}

Foi desenvolvido e testado um método para avaliação do comportamento catalítico de pelotas/DRI's quanto a reação de reforma do metano nas condições dos processos de redução direta.

Em condições não carburantes e na faixa de temperatura de $875{ }^{\circ} \mathrm{C}$ a $1050{ }^{\circ} \mathrm{C}$, a etapa controladora da reação de reforma de metano até conversões de metano de 0,50 é a reação química na interface entre o gás e a superfície do ferro reduzido do DRI. O mecanismo proposto por Münster e Grabke ${ }^{(3,4)}$ explica bem os resultados obtidos até este nível de conversão. A energia de ativação obtida foi de $258 \mathbf{~ k J} / \mathbf{m o l}$.

O mecanismo de controle da cinética em conversões de metano acima de 0,50 ainda não está determinado.

A utilização de composição de gás de entrada no limite das condições de carburação $\left(\mathrm{H}_{2} \mathrm{O} / \mathrm{CH}_{4}=1\right)$ gerou a carburação das amostras de ferro, mas com consumo de $2,0 \%$ ou menos do volume de metano injetado.

Em condições carburantes $\left(\mathrm{H}_{2} \mathrm{O} / \mathrm{CH}_{4}=0,25\right)$, a carburação do ferro é intensa consumindo $30 \%$ do metano injetado e levando a teores de carbono no DRI acima do contido no $\mathrm{Fe}_{3} \mathrm{C}$. Isto compromete a atuação do $\mathrm{DRI}$ como catalisador da reação de reforma, uma vez que o gás passa a reagir com o carbono sólido depositado.

\section{Agradecimentos}

Os autores agradecem pelo auxílio oferecido pelo projeto GASFERROSIL (número da concessão 224950/E30) financiado pelo Conselho de Pesquisa da Noruega e pela FIPT (Fundação de Apoio ao Instituto de Pesquisas Tecnológicas).

\section{REFERÊNCIAS}

1 Midrex Technology Inc. 2015 World Direct Reduction Statistics. Charlotte, NC, EUA 2016.

2 A. Martins, D. D. Nogare, A. Volpatti, P. Duarte. High-Carbon Hot DRI Production and USE: Energiron Results and Performances in Operating Plants", Proceedings of the AISTech 2015, Cleveland, OH, EUA, 4-7 Maio, 2015.

3 Münster, P.; Grabke, H.J. Kinetics of the Steam Reforming of Methane on Iron as a Catalyst. Berichte der Bunsengesellschaft für Physikalische Chemie, vol 84, ed.10, out/1980, p. 1068-1071.

4 Münster, P.; Grabke, H.J. Kinetics of the steam reforming of methane with iron, nickel, and iron-nickel alloys as catalysts. Journal of Catalysis. vol. 72, ed. 2, dez/1981, p.279_ 287

5 Fogler, H.S. Elements of Chemical Reaction Engineering. $3^{a}$ edição, Prentice Hall, New Jersey, EUA, 200.

6 Ribeiro, T.R.; Ferreira Neto, J.B.; Poço, J.G.R.; Kolbeinsen, L.; Ringdalen, E. Methane reforming catalyzed by Direct Reduced Iron. Anais da conferência AISTech17, AIST, Nashville, TN, EUA, 08 a 11 de maio de 2017. 